\title{
Approaches towards tick and tick-borne diseases control
}

\author{
Ana Domingos ${ }^{[1]}$, Sandra Antunes ${ }^{[2]}$, Lara Borges $^{[1]}$ and Virgílio Estólio do Rosário ${ }^{[2]}$
}

[1]. Centro de Malária e outras Doenças Tropicais, Instituto de Higiene e Medicina Tropical, Lisboa, Portugal. [2]. Instituto de Higiene e Medicina Tropical, Lisboa, Portugal.

\begin{abstract}
Ticks are obligate haematophagous ectoparasites of wild and domestic animals as well as humans, considered to be second worldwide to mosquitoes as vectors of human diseases. Tick-borne diseases are responsible worldwide for great economic losses in terms of mortality and morbidity of livestock animals. This review concerns to the different tick and tick-parasites control methods having a major focus on vaccines. Control of tick infestations has been mainly based on the use of acaricides, a control measure with serious drawbacks, as responsible for the contamination of milk and meat products, as a selective factor for acaricideresistant ticks and as an environmental contaminant. Research on alternatives to the use of acaricides is strongly represented by tick vaccines considered a more cost-effective and environmentally safe strategy. Vaccines based on the Bm86 tick antigen were used in the first commercially available cattle tick vaccines and showed good results in reducing tick numbers, affecting weight and reproductive performance of female ticks which resulted in reduction of cattle tick populations over time and consequently lower reduction of the pathogen agents they carry.
\end{abstract}

Keywords: Ticks. Tick-borne diseases. Control methods. Vaccines.

\section{TICKS AND TICK-BORNE DISEASES}

Ticks are arthropods with a complex life cycle, proven resilient and persistent in the environment. Three families are currently recognized: Ixodidae (hard ticks), Argasidae (soft ticks) and the recently identified Nuttalliellidae ${ }^{1}$. Hard ticks distinguish themselves by the presence of a scutum or hard shield that grows to accommodate large volumes of ingested blood, which, in adult ticks, reaches 200 to 600 times their unfed body weight. On the contrary, soft ticks accommodate smaller volumes of blood ( 5 to 10 times their unfed body) and can resist to starvation, surviving for long periods of time without a blood meal $^{2}$. The Nuttalliellidae family contains only one species, Nuttalliela namaqua, which exhibits intermediate characteristics in comparison with the other two ${ }^{1}$. Adult ticks, larvae or nymphs (pre-adult stages) can be infected horizontally by feeding on infected vertebrate hosts, or within the ticks, from the female to the eggs, maintaining the infection to the hatched larvae, a phenomenon denoted as transovarial transmission. There is also the transtadial transmission, which is the pathogen's diffusion from one tick life stage through a molt to the next instar ${ }^{3}$.

These ecto-parasites have direct impact on the vertebrate hosts, leading to reduction of body weight, and in cattle, affect the milk and meat production, while ticks' bites reduce the quality of leathers. Among domestic animals, they are also responsible for anemia and severe dermatitis ${ }^{4-6}$. Apart from these direct effects, the most important feature of ticks is that they are vectors, as well as reservoirs, of multiple pathogens. Ticks and tick-transmitted parasites have co-evolved with various

Address to: Dra. Ana Domingos. IHMT/UNL. Rua da Junqueira 100, 1349-008 Lisboa, Portugal.

Phone: 35121 365-2600; Fax: 35121 363-2105

e-mail: adomingos@ihmt.unl.pt

Received 01 October 2012

Accepted 11 December 2012 wild animal hosts, being part of the ecosystem's equilibrium ${ }^{3}$. Tick-borne diseases (TBDs), long known but often neglected, are progressively being recognized due to their economic impact in livestock, but also due to their impact in human health, to which they have become a threat.

Examples of TBDs transmitted to man are Lyme borreliosis by Ixodes sp. ticks, caused by at least three species of bacteria belonging to the genus Borrelia spp. and Rocky Mountain spotted fever, caused by Rickettsia rickettsii spread by Dermacentor variabilis. Changes in land use, reforestation, human demographics and behavior, are altering the interactions between human and infectious disease agents leading to the emergence of other infectious and zoonotic diseases ${ }^{7}$. Under these circumstances, arthropod vectors may enhance their potential to spread bacteria, viruses, protozoa and helminthes ${ }^{8}$. Nevertheless, the major medical and economic concerns with ticks and TBDs remain in the veterinarian field with a special emphasis on animal production. TBDs, as theileriosis, babesiosis, anaplasmosis and heartwater (also called cowdriosis), are considered the most important, concerning both health and management problems of cattle and small ruminants, especially in Latin America, Africa, Australia and Asia. Economically, the most important livestock ticks belong to the family Ixodidae, genera Hyalomma spp., Rhipicephalus (Boophilus) spp. and Amblyomma spp. Theileriosis and babesiosis are caused by the protozoan parasites Theileria parva, T. annulata and Babesia bovis or B. bigemina, correspondingly. While the heartwater and anaplasmosis are caused by the Rickettsiales, Ehrlichia ruminantium and Anaplasma marginale, respectively ${ }^{2,8,9}$.

\section{CONTROL METHODS}

So far, the use of acaricides has been a major component of integrated tick control methods. Even before Smith \& Kilborne (1893) proved the role of ticks as vectors of Babesia spp., animal 
health authorities in the USA, Australia and Southern Africa were treating cattle with a variety of chemical agents, mainly mixtures of querosene, sulphur and lard, in an effort to control ticks. Acaricides are often inappropriately used, have residual effects in milk and meat subproducts, and are not environmentally friendly, being responsible for the increase of acaricide-resistant ticks ${ }^{10,11}$. Resistance is associated to mutations in genes related to drug susceptibility. The appearance of acaricides' resistance leads to the rise of individuals for which the lethal dose is higher than the one for the majority of determined specie. Nowadays, combinations of powerful acaricides are being used worldwide; products combining different active components are available in an attempt to include a diverse number of mechanisms of action, to reduce the emergence of insecticide resistance ${ }^{12,13}$.

For companion animals, a formulation combining dinotefuran, permethrin and pyriproxyfen (Vectra 3D) was registered in the USA in 2007, and is indicated for the prevention and treatment of fleas, ticks, flies and mosquitoes, on dogs ${ }^{14,15}$. Others like imidacloprid/flumethrin collar [Seresto ${ }^{\circledR}$, Bayer Animal Health, Investigational Veterinary Product (IVP)], a deltamethrin collar (Scalibor ${ }^{\circledR}, \mathrm{MSD}, \mathrm{CP} 1$ ), a fipronil/(s)-methoprene spot-on (Frontline Combo®, Merial, CP2), and an amitraz/fipronil/ (s)-methoprene spot-on (Certifect $\AA$, Merial, CP4/CP5) against repeated infestations with Rhipicephalus sanguineus and Ctenocephalides felis felis are being tested for efficacy ${ }^{15}$. The example of pet animals is not valid in the scenario of animal production. George et al. offers an extended review on chemical control of ticks that can be consulted for further information ${ }^{10}$.

Recent studies in Brazil and Mexico showed that the resistance to drugs such as cipermetrine and amitraz in Rhipicephalus microplus, and other ticks, is increasing ${ }^{13,16-18}$. The speed, with which resistance has appeared, along with the significantly more expensive pesticides, has restrained the companies to develop new drugs. The introduction of a new product in the market is time-consuming and has a huge economic burden; being the cost of discovering and developing a novel product estimated in US\$100 million, with an average duration of 10 years $^{11}$. This and the increasing concerns about resistance and side effects of insecticidal compounds, has led to the introduction of few new products over the years (e.g. spinosad) $)^{12,19}$.

Recently, particular attention has been focused on the development of entomopathogenic fungi ${ }^{20}$, such as Metarhizium anisopliae and Beauveria bassiana, as biocontrol agents against a range of several ticks under laboratory and field conditions, namely Rhipicephalus annulatus ${ }^{21}$, Ixodes scapularis $^{22}$, Rhipicephalus appendiculatus and Amblyomma variegatum $^{23}$, Argas persicus ${ }^{24}$ and Ornithodoros lahorensis ${ }^{25}$. Biocontrol agents usually favor both human and environmental safety, especially in comparison to the use of acaricides, but few biopesticide have been used in spite of their potential. The inability to successfully adopt biocontrol strategies includes factors like environmental stability (e.g., UV resistance, temperature tolerance), ability to initiate infection at low humidity, and potential unspecific damage to non-target invertebrates ${ }^{24,25}$.
In this review the focus is on the new strategies becoming available for the control of ticks and associated pathogens in cattle.

\section{VACCINES}

Globally, most of the vaccines available to overcome TBDs are attenuated or live blood-derived. In theileriosis, the pathogens' life cycle involves three developmental stages: sporozoites, schizonts and piroplasms ${ }^{26}$. Cattle that recover from infection with Theileria parva or Theileria annulata are solidly protected against subsequent infections with homologous strains but can succumb to heterologous challenge. Immunization with simple mixtures of parasite strains results in an attenuated infection that produces an effective immune response ${ }^{27,28}$. The only commercialized $T$. annulata vaccine is based on attenuated shizonts produced in cell culture (Rak-shavac-T®, National Dairy Development Board, India). Cattle immunization with sporozoite surface antigen-1 or attenuated schizont-infected cells induces limited protection against homologous or heterologous sporozoite challenge, whereas a combination of recombinant and live vaccine results in survival of all vaccinates ${ }^{29}$. Attenuated vaccines have also been used to protect cattle against babesiosis and anaplasmosis, being these results evidence for the creation of improved immunity, by including sporozoite and schizont antigens in vaccines. Attenuated vaccines have successfully been used against babesiosis for example in Argentina, Israel and Australia. Moreover, Australian Babesia bovis and Babesia bigemina attenuated strains are being used to immunize cattle in other regions of the world namely, Africa South America and South-East Asia as described in Office of Environmental Information (OEI) report ${ }^{30}$. Though these vaccines can be effective, little is known about their full mechanism of action. They comprise important drawbacks, as a short shelf life, the potential transmission of other pathogens and the possible reversion to virulence, requiring a cold chain system of maintenance. Therefore, an additional research is needed for the development of safer alternatives, more cost-effective and better defined live, or subunit, vaccines. Nonetheless, alternative approaches have been raised to control TBDs, which involve the development of anti-tick vaccines that can quell both vector and pathogens ${ }^{8,31}$.

Recombinant vaccines became commercially available in the early 1990s, aiming to reduce the use of acaricides and their consequences. The feasibility of controlling tick infestations through immunization of hosts with selected tick antigens was achieved developing vaccines that reduced infestations on cattle. Vaccines against ticks allowing the inclusion of multiple antigens that could target a broad range of tick species and could also prevent transmission of pathogens ${ }^{32}$.

Tick antigens are usually regarded as either exposed or concealed antigens. Exposed antigens are those that naturally come into contact with the host immune system during tick infestation. Hosts immunized with these antigens are boosted by continuous tick exposure. Concealed antigens are not exposed to the host immune system and therefore repeated 
immunizations are required to maintain high antibody titers. However, concealed antigens are more advantageous once ticks are unlikely to have evolved a mechanism to counteract the host immune response, contrarily to an exposed antigen ${ }^{32,33}$.

A great handicap in the development of anti-tick vaccines, like other anti-parasite vaccines, is the identification of effective antigens. One of the major constraints when working with obligate intracellular parasites is a large excess of proteins of host or vector origin that interfere with pathogen protein detection. Among the important characteristics for a concealed antigen is the accessibility to antibody ingested during tick feeding and a vital physiological function of the tick ${ }^{32}$. The advances in characterization of tick genomes, along with the use of bioinformatics, ribonucleic acid interference (RNAi), mutagenesis, immunomapping, transcriptomics, proteomics, expression library immunization (ELI) and other technologies has allowed a rapid, systematic and comprehensive approach to tick vaccine discovery ${ }^{32}$.

An effective antigen against ticks is the protein $\mathrm{Bm} 86$, specifically directed against the cattle tick Rhipicephalus microplus, it stands as the basis of two commercial vaccines, TickGARD Plus and Gavac Plus. The greatest effect was the reduction of larval infestations in subsequent generations, by reducing the number of engorging female ticks, their weight, and reproductive capacity $19,31,34,35$.

Pipano et al. tested the efficacy of a Bm86 vaccine in protection against ticks and pathogens transmitted by those ectoparasites $(B \text {. bovis and B. bigemina })^{36}$. The results showed that immunized cattle, when challenged with $B$. bovis-infected ticks, continued to become infected, but in the case of B. bigemina, Bm86-immunized animals remained protected against infection ${ }^{36}$. Canales et al. have cloned ortholog genes (Ba86 and Bm86) from $R$. annulatus and $R$. microplus, respectively ${ }^{37}$. Cattle vaccination with $\mathrm{Bm} 86$ reduced the $R$. annulatus and $R$. microplus numbers, weight, oviposition and eggs' fertility. For Rhipicephalus decoloratus, Odongo et al., using a $\mathrm{Bm} 86$ based-vaccine, found a reduction on engorged adult female ticks, ticks weight and eggs weight ${ }^{38}$. Bastos, et al. studied the Bm86 silencing on the ability of $R$. microplus ticks to feed in $B$. bovis infected cattle, showing that this procedure decreased survival engorged ticks rate and eggs weight ${ }^{39}$.

Rhipicephalus microplus Bm86/Bm95 antigens have proven their efficacy for the control of cattle tick infestations and transmission of tick-borne pathogens but only in some regions. In fact, the 900 tick species that have been documented are distributed by several geographic areas and animals are, therefore exposed to different tick stocks ${ }^{40,41}$. Several approaches have been made, like double vaccination with different activeprinciples and/or several doses along the time ${ }^{42,43}$, and it became clear that for every region and tick species, should be formulated a different immunization procedure. Nonetheless, this approach is highly expensive and technically challenging ${ }^{9,31}$.

Some examples of research studies aiming the identification of new vaccines are here described. The protein 64P from $R$. appendiculatus was found to be involved in ticks attachment and feeding, and was used to immunize guinea pigs, reducing nymph and adult infestation ${ }^{44}$. First studies on yolk pro-Cathepsin expressed in eggs of $R$. microplus suggested this aspartic proteinase as a promising antigen, however, when expressed as a recombinant protein in Escherichia coli and tested in a cattle trial, the efficacy was merely $25 \%{ }^{45}$. Another example concerns to 5 '-nucleotidase that when tested in sheep showed positive results, but no effect in a subsequent trial in cattle ${ }^{36}$. The gut-expressed iron storage protein, ferritin 2, is another antigen that has been evaluated in cattle trials. Silencing of ferritin 2 by RNA interference showed significant impacts on tick feeding, oviposition and larval hatch, indicating ferritin 2 as a candidate tick vaccine antigen ${ }^{46}$.

Subolesin, first discovered in I. scapularis, is a highly conserved protein involved in modulation of tick feeding and reproduction, and had a protective effect against all tick developmental stages when used in recombinant protein immunization. Subolesin was silenced by de la Fuente et al. through RNAi in D. variabilis, leading to degeneration of several tick tissues, such as guts, salivary glands, reproductive tissues and embryos ${ }^{32}$. Therefore, production of sterile ticks was made possible through subolesin knockdown by RNAi. Consequently, the release of subolesin-silenced ticks, as a sterile acarine technique (SAT), for autocidal control of tick populations has been proposed ${ }^{43}$. By releasing enough numbers of sterile individuals that mate with wild ones, it is expected a decrease in the wild population overtime, due to the lowering of the reproductive potentia ${ }^{48}$. The use of sterile insect technique has proved its utility in dealing with crop pests, but its potential in tick control has not yet been explored in a larger scale. Kocan et al. showed that subolesin knockdown in I. scapularis, Dermacentor variabilis and Amblyomma americanum also affected oviposition, eggs embryogenesis, larval hatching and fertility ${ }^{47}$. Vaccination with subolesin reduced $R$. microplus survival and reproduction rates and tick infection by Anaplasma spp. and Babesia spp. ${ }^{41}$. Their results demonstrated that $R$. microplus infestations where successfully controlled by combining vaccination and release of transgenic ticks, which suggests that the combination of methods increases the efficacy of cattle tick control, at least under some circumstances. Further studies are being developed concerning cattle vaccination in different regions of the world, to determine whether the promising results obtained in Mexico can be reproduced elsewhere ${ }^{8,9,42}$.

Looking for new antigens, Antunes et al. have characterized $R$. annulatus genes differentially expressed in response to $B$. bigemina infection using suppression-subtractive hybridisation (SSH) and real-time reverse transcription polymerase chain reaction (RT-PCR). Genes confirmed as differentially expressed in infected ticks [tick receptor for OspA (TROSPA), calreticulin, ricinusin, serum amyloid A and Kunitz-type protease inhibitor (KTPI)] were functionally characterized using RNAi approach to analyze their role during pathogen infection in the tick vector ${ }^{49}$.

Pal et al. had already described TROSPA in I. scapularis, studying the effect of anti-TROSPA antibodies and gene knockdown during a $B$. burgdorferi infection. Reduced $B$. burgdorferi adherence to the I. scapularis gut was observed 
in vivo, culminating in a deficient colonization of the vector, with subsequent levels reduction of pathogens transmission to the mammalian host ${ }^{50}$. Antunes et al. found a similar protein over-expressed in B. bigemina-infected $R$. annulatus, with high sequence identity to Ixodes spp. After TROSPA knockdown, significant decrease in infection was observed for both $R$. annulatus and $R$. microplus. These results suggested the possibility that $B$. bigemina uses a TROSPA ortholog receptor for infection of Rhipicephalus tick cells and encouraged research for the characterization of this molecule in Babesia-tick interactions and development of transmission blocking vaccines. Serum amyloid A is known for being involved in host response to tissue injury and inflammation. After gene knockdown in both Rhipicephalus spp. lower infections were observed without weight losses, suggesting that this protein may be part of tick response to the stress produced by Babesia sp. infection, but at the same time necessary for pathogen multiplication in Rhipicephalus spp. ticks ${ }^{49}$. Calreticulin, already described in tick's saliva by Jaworski et al. and Ferreira et al., seems to take a role during tick feeding, once gene knockdown resulted in a weight decrease of $R$. annulatus ${ }^{51,52}$. These results also suggest that this protein may be required for B. bigemina infection. Gene knockdown of ricinusin did not affect pathogen infection, thus suggesting that this molecule is not essential to control B. bigemina infection in Rhipicephalus spp. ticks ${ }^{49}$.

The absence of full tick genomic data and the lack of a confirmed tick RNAi pathway can underestimate the off-target effects in current tick RNAi experiments ${ }^{53}$. Despite this, the use of long dsRNAs as gene knockdown treatments in ticks has been accepted as a routine method for validation/support of tick gene function $^{42,54,55}$. Some of the $R$. annulatus genes discovered in this study such as serum amyloid A, calreticulin and TROSPA could contribute to the development of novel vaccines designed to reduce tick infestations and prevent or minimize pathogen infection in ticks and transmission to vertebrate hosts ${ }^{49}$.

\section{CONCLUSIONS}

These revision concerns to a discussion on the methods used for tick and tick-borne parasites control and was mainly focused on the development of new recombinant vaccines.

Research in the post-genomic era is leading to the development of new control measures such as the recombinant vaccines. Despite these advances the establishment of non-living vaccines has been challenging. As a result, and despite several disadvantages, attenuated vaccines are still being used, adapted to conditions of each region.

After more than a decade, the two commercial tick recombinant vaccines are still being used in some countries such as Cuba, Australia and Mexico, though not worldwide due to commercial and technical constrains. These vaccines, however, when used in field trials, showed very positive results on tick and tick-borne diseases (TBD) reduction, improving cattle production and reducing dependency on acaricides. In parallel, they also showed to be a cost-effective and environmentally safe strategy, to tick control.
Tick infestation is rarely a one-species issue, and therefore, anti-tick vaccines should aim at a more global protection against the main species of economical and epidemiological interest. The great rise of acaricide resistance, still asks for the implementation of an effective vaccine. The discovery of potential antigens against tick and tick-pathogens proteins should result in improved vaccines, more advantageous in an overall approach to control TBDs.

\section{CONFLICT OF INTEREST}

The authors declare that there is no conflict of interest.

\section{REFERENCES}

1. Guglielmone AA, Robbing RG, Apanaskevich DA, Petney TN, Estrada-Peña A, Horak IG, et al. The Argasidae, Ixodidae and Nuttalliellidae (Acari: Ixodida) of the world: a list of valid species names. Zootaxa 2010; 2528:1-28.

2. Rajput ZI, Hu S, Chen W, Arijo AG, Xiao C. Importance of ticks and their chemical and immunological control in livestock. J Zhejiang Univ Science B 2006; 7:912-921.

3. Jongejan F, Uilenberg G. The global importance of ticks. Parasitol 2004; 129:S3-S14.

4. Food and Agriculture Organization of the United Nations (FOA) [Internet]. FOA;1998. [Cited 2012 July 15] Available from: http://www.fao.org/ag/AGA/ AGAH/PD/pages/tick01.htm/.

5. L'Hostis M, Seegers H. Tick-borne parasitic diseases in cattle: current knowledge and prospective risk analysis related to the ongoing evolution in French cattle farming systems. Vet Res 2002; 33:599-611.

6. Peter RJ, van den Bossche P, Penzhorn BL, Sharp B. Tick, fly, and mosquito control lessons from the past, solutions for the future. Vet Parasitol $2005 ; 132: 205-215$.

7. Douglas D. Colwell, Filipe Dantas-Torres, Domenico Otranto. Vector-borne parasitic zoonoses: Emerging scenarios and new perspectives. Vet Parasitol 2011; 182:14-21.

8. Guerrero FD, Miller RJ, Pérez de León AA. Cattle tick vaccines: Many candidate antigens, but will a commercially viable product emerge? Int J Parasitol 2012; 42:421-427.

9. Parizi LF, Githaka NW, Logullo C, Konnai S, Masuda A, Ohashi K, et al. The quest for a universal vaccine against ticks: Cross-immunity insights. Vet J 2012; 194:158-165.

10. George JE, Pound JM, Davey RB. Chemical control of ticks on cattle and the resistance of these parasites to acaricides. Parasitol 2004; 129:353-366.

11. Graf JF, Gogolewski R, Leach-Bing N, Sabatini GA, Molento MB, Bordin EL, et al. Tick control: an industry point of view. Parasitol 2004; 129:427-442.

12. Gentz MC, Murdoch G, King GF. Tandem use of selective insecticides and natural enemies for effective, reduced-risk pest management. Biol Control 2010; 52:208-215.

13. Veiga LP, Souza AP, Bellato V, Sartor AA, Nunes AP, Cardoso HM. Resistance to cypermethrin and amitraz in Rhipicephalus (Boophilus) microplus on the Santa Catarina Plateau, Brazil. Rev Bras Parasitol Vet 2012; 21:133-136.

14. Franc M, Genchi C, Bouhsira E, Warin S, Kaltsatos V, Baduel L, et al. Efficacy of dinotefuran, permethrin and pyriproxyfen combination spot-on against Aedes aegypti mosquitoes on dogs. Vet Parasitol 2012; 189:333-337.

15. Horak IG, Fourie JJ, Stanneck D. Efficacy of slow-release collar formulations of imidacloprid/flumethrin and deltamethrin and of spot-on formulations of fipronil/ (s) - methoprene, dinotefuran/pyriproxyfen/permethrin and (s) - methoprene/ amitraz/fipronil against Rhipicephalus sanguineus and Ctenocephalides felis felis on dogs. Parasit Vectors 2012; 5:79.

16. Dantas-Torres F. The brown dog tick, Rhipicephalus sanguineus (Latreille, 1806) (Acari: Ixodidae): from taxonomy to control. Vet Parasitol 2008; 152:173-185.

17. Guerrero FD, Soares MA, Barros JC, Miller RJ, Léon AP. Acaricide resistance of Rhipicephalus (Boophilus) microplus in State of Mato Grosso do Sul, Brazil. Rev Bras Parasitol Vet 2011; 20:127-133. 
18. Fernández-Salas A, Rodriguez-Vivas RI, Alonso Díaz MA. Resistance of Rhipicephalus microplus to amitraz and cypermethrin in tropical cattle farms in Veracruz, Mexico. J Parasitol 2012; 98:1010-1014.

19. Willadsen P. Anti-tick vaccines. Parasitol 2004; 129:367-387.

20. Hajek AE, Delalibera Jr I. Fungal pathogens as classical biological control agents against arthropods. BioControl 2012; 55:147-158.

21. Pirali-Kheirabadi K, Haddadzadeh H, Razzaghi-Abyaneh M, Bokaie S, Zare R, Ghazavi M, et al. Biological control of Rhipicephalus (Boophilus) annulatus by different strains of Metarhizium anisopliae, Beauveria bassiana and Lecanicillium psalliotae fungi. Parasitol Res 2007; 100:1297-1302.

22. Hornbostel VL, Ostfeld RS, Benjamin MA. Effectiveness of Metarhizium anisopliae (Deuteromycetes) against Ixodes scapularis (Acari: Ixodidae) engorging on Peromnyscus leucopus. J Vector Ecol 2005; 30:91-101.

23. Kaaya GP, Mwangi EN, Ouna EA. Prospects for biological control of livestock ticks, Rhipicephalus appendiculatus and Amblyomma variegatum, using the entomogenous fungi Beauveria bassiana and Metarhizium anisopliae. J Invertebr Pathol 1996; 67:15-20.

24. Pourseyed SH, Tavassoli M, Bernousi I, Mardani K. Metarhizium anisopliae (Ascomycota: Hypocreales): an effective alternative to chemical acaricides against different developmental stages of fowl tick Argas persicus (Acari: Argasidae). Vet Parasitol 2010; 172:305-310.

25. Tavassoli M, Malekifard F, Soleimanzadeh A, Pourseyed SH, Bernousi I, Mardani K. Susceptibility of different life stages of Ornithodoros lahorensis to entomopathogenic fungi Metarhizium anisopliae and Beauveria bassiana. Parasitol Res 2012; 111:1779-1783.

26. Preston PM, Hall FR, Glass EJ, Campbell JD, Darghouth MA, Ahmed JS, et al. Innate and adaptive immune responses co-operate to protect cattle against Theileria annulata. Parasitol Today 1999; 15:268-274.

27. Radley DE, Brown CGD, Cunningham MP, Kimber CD, Musisi FL, Payne RC, et al. East Coast fever: 3. Chemoprophylactic immunization of cattle using oxytetracycline and a combination of theilerial strains. Vet. Parasitol 1975; 1:51-60.

28. Pipano E, Shkap V. Vaccination against tropical theileriosis. Ann NYAcad Sci 2000; 916:484-500.

29. Darghouth MA, Boulter NR, Gharbi M, Sassi L, Tait A, Hall R.Vaccination of calves with attenuated cell line of Theileria annulata and the sporozoite antigen SPAG-1 produces a synergistic effect. Vet Parasitol 2006; 142:54-62.

30. World organisation for animal health (Oie). Manual of Diagnostic Tests and Vaccines for Terrestrial Animals [Internet]. Oie; 2012. [Cited 2012 July 25] Available from: http://www.oie.int/manual-of-diagnostic-tests-and-vaccinesfor-terrestrial-animals/

31. Marcelino I, Almeida AM, Ventosa M, Pruneau L, Meyer DF, Martinez D, et al. Tick-borne diseases in cattle: Applications of proteomics to develop new generation vaccines. J Proteomics 2012; 75:4232-4250.

32. Fuente J, Kocan KM. Strategies for development of vacines for control of ixodid tick species. Parasite Immunol 2006; 28:275-283.

33. Kiss T, Cadar D, Spînu M. Tick prevention at a crossroad: New and renewed solutions. 2012. Vet Parasitol 2012; 187: 357-366.

34. Almazán C, Lagunes R, Villar M, Canales M, Rosario-Cruz R, Jongejan F, et al. Identification and characterization of Rhipicephalus (Boophilus) microplus candidate protective antigens for the control of cattle tick infestations. Parasitol Res 2010; 106:471-479.

35. Hope M, Jiang X, Gough J, Cadogan L, Josh P, Jonsson N, et al. Experimental vaccination of sheep and cattle against tick infestation using recombinant 5-nucleotidase. Parasite Immunol 2012; 32:135-142.

36. Pipano E, Alekceev E, Galker F, Fish L, Samish M, Shkap V. Immunity against Boophilus annulatus induced by the Bm86 (Tick-GARD) vaccine. Exp Appl Acarol 2003; 29:141-149.

37. Canales M, Almazán C, Naranjo V, Jongejan F, Fuente J. Vaccination with recombinant Boophilus annulatus $\mathrm{Bm} 86$ ortholog protein, $\mathrm{Ba} 86$, protects cattle against B. annulatus and B. microplus infestations. BMC Biotechnol 2009; 9:29.
38. Odongo D, Kamau L, Skilton R, Mwaura S, Nitsch C, Musoke A, et al. Vaccination of cattle with TickGARD induces cross-reactive antibodies binding to conserved linear peptides of Bm86 homologues in Boophilus decoloratus. Vaccine 2007; 25:1287-1296.

39. Bastos RG, Ueti MW, Knowles DP, Scoles GA. The Rhipicephalus (Boophilus) microplus Bm86 gene plays a critical role in the fitness of ticks fed on cattle during acute Babesia bovis infection. Parasit Vectors 2010; 3:111.

40. Fuente J, Almazán C, Canales M, Pérez de la Lastra JM, Kocan KM, Willadsen P. A ten-year review of commercial vaccine performance for control of tick infestations on cattle. Anim Health Res Rev 2007; 8:23-28.

41. Merino O, Almazan C, Canales M, Villar M, Moreno-Cid JA, Galindo RC, et al. Targeting the tick protective antigen subolesin reduces vector infestations and pathogen infection by Anaplasma marginale and Babesia bigemina. Vaccine $2011 ; 29: 8575-8579$.

42. Merino O, Almazán C, Canales M, Villar M, Moreno-Cid JA, Estrada-Peña A, et al. Control of Rhipicephalus (Boophilus) microplus infestations by the combination of subolesin vaccination and tick autocidal control after subolesin gene knockdown in ticks fed on cattle. Vaccine 2011; 29:2248-2254.

43. Shkap V, Vos AJ, Zweygarth E, Jongejan F. Attenuated vaccines for tropical theileriosis, babesiosis and heartwater: the continuing necessity. Trends Parasitol 2007; 23:420-426.

44. Trimnell AR, Hails RS, Nuttall PA. Dual action ectoparasite vaccine targeting 'exposed' and 'concealed' antigens. Vaccine 2002; 20:3560-3568.

45. Leal AT, Seixas A, Pohl PC, Ferreira CA, Logullo C, Oliveira PL, et al. Vaccination of bovines with recombinant Boophilus yolk pro-cathepsin. Vet Immunol Immunopathol 2006; 114:341-345.

46. Hajdusek O, Almazán C, Loosova G, Villar M, Canales M, Grubhoffer L, et al. Characterization of ferritin 2 for the control of tick infestations. Vaccine 2012; 28:2993-2998.

47. Sparagano O, Luna CJ. From population structure to genetically-engineered vectors: New ways to control vector-borne diseases? Infect Genet Evol 2008; 8:520-525.

48. Kocan K, Manzano-Roman R, Fuente J. Transovarial silencing of the subolesin gene in three-host ixodid tick species after injection of replete females with subolesin dsRNA. Parasitol Res 2007; 100:1411-1415.

49. Antunes S, Galindo RC, Almazán C, Rudenko N, Golovchenko M, Grubhoffer L, et al. Functional genomics studies of Rhipicephalus (Boophilus) annulatus ticks in response to infection with the cattle protozoan parasite, Babesia bigemina. Int J Parasitol 2012; 42:187-195.

50. Pal U, Li X, Wang T, Montgomery RR, Ramamoorthi N, Desilva AM, et al. TROSPA, an Ixodes scapularis receptor for Borrelia burgdorferi. Cell 2004; 119:457-468.

51. Jaworski DC, Simmen FA, Lamoreaux W, Lewis B, Coons LB, Muller MT, et al. A secreted calreticulin protein in Ixodid tick (Amblyomma americanum) saliva. J Insect Physiol 1995; 41:369-375.

52. Ferreira CA, Silva-Vaz I, Silva SS, Haag KL, Valenzuela JG, Masuda A. Cloning and partial characterization of a Boophilus microplus (Acari: Ixodidae) calreticulin. Exp Parasitol 2002; 101:25-34.

53. Lew-Tabor AE, Kurscheid S, Barrero R, Gondro C, Moolhuijzen PM, Rodriguez-Valle M, et al. Gene expression evidence for off-target effects caused by RNA interference-mediated gene silencing of Ubiquitin-63E in the cattle tick Rhipicephalus microplus. Int J Parasitol 2011; 41:1001-1014.

54. Fuente J, Blouin EF, Manzano-Roman R, Naranjo V, Almazán C, Pérez de la Lastra JM, et al. Functional genomic studies of tick cells in response to infection with the cattle pathogen, Anaplasma marginale. Genomics 2007; 90:712-722.

55. Smith A, Guo X, Fuente J, Naranjo V, Kocan KM, Kaufman WR. The impact of RNA interference of the subolesin and voraxin genes in male Amblyomma hebraeum (Acari: Ixodidae) on female engorgement and oviposition. Exp Appl Acarol 2009; 47:71-86. 